\title{
Mitteilungen der DGTHG
}

Z Herz-Thorax- Gefäßchir 2017 · 31:438

https://doi.org/10.1007/s00398-017-0191-9

(๑) Springer Medizin Verlag GmbH, ein Teil von Springer Nature 2017

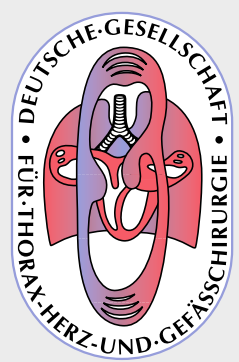

Deutsche Gesellschaft für

Thorax-, Herz- und Gefäßchirurgie (DGTHG)

Geschäftsstelle

Langenbeck-Virchow-Haus

Luisenstr. 58/59

10117 Berlin

Tel. 030/28004370

Fax 030/28004379

sekretariat@dgthg.de

www.dgthg.de

Dr. A. Beckmann (V.i.S.d.P.)

Geschäftsführer der DGTHG

\section{Ankündigung der Jahrestagung 2018}

Die 47. Jahrestagung der Deutschen Gesellschaft für Thorax-, Herzund Gefäßchirurgie wird vom 17.-20. Februar 2018 in Leipzig stattfinden, zeitgleich mit der Jahrestagung der Deutschen Gesellschaft für Pädiatrische Kardiologie.

\section{Sekretär der DGTHG}

Prof. Dr. Andreas Markewitz

DGTHG, Langenbeck-Virchow-Haus

Luisenstraße 58/59

10117 Berlin

Tel: 030/28004370

Fax: 030/28004379

E-Mail: sekretaer@dgthg.de

\section{Einladung}

Alle Mitglieder der Deutschen Gesellschaft für Thorax-, Herz- und Gefäßchirurgie (DGTHG) sind zur Ordentlichen Mitgliederversammlung eingeladen. Diese findet im Rahmen der Jahrestagung statt:

Montag, 19. Februar 2018, 16.00-17.30 Uhr,

Mehrzweckraum C, Leipziger Messe.

\section{Vorläufige Informationen zu den Wahlen:}

Folgende Vorstandspositionen müssen 2018 per Wahl neu besetzt werden:

Drei (3) Beisitzer

Der Vorstand schlägt zur Wiederwahl (2. Amtsperiode) vor:

- Prof. Dr. Johannes Albes

Der Vorstand schlägt zur Neuwahl vor:

- Prof. Dr. Sabine Bleiziffer

- Prof. Dr. Matthias Siepe
Weitere Wahlvorschläge sind bis zum 19. Januar 2018 an das Sekretariat der DGTHG zu richten:

E-Mail: sekretariat@dgthg.de, Fax: +49-30-28004379.

Satzungsgemäß wird die offizielle Einladung zur Mitgliederversammlung zusammen mit der Tagesordnung mindestens zwei Wochen im Voraus versandt werden.

\section{Neue Mitglieder}

\section{Ordentliche Mitglieder}

- Alaedin ADDAS, Herzzentrum Leipzig

- Lennart BAX, Universitäres Herzzentrum, Hamburg

- Dr. med. Asmae GASSA, Universitätsklinik Köln

- Florian HECKER, Kerckhoff Klinik, Bad Nauheim

- Adela LAZAR, Helios Klinik, Karlsruhe

- Dr. med. Ajay MOZA, Universitätsklinik RWTH, Aachen

- Dr. med. Jonas PAUSCH, Universitäres Herzzentrum, Hamburg

- Johanna RICHAU, Herz und Gefäßchirurgie, Klinikum Coswig

- Konstantin ZHIGALOV, Klinikum Oldenburg 